\title{
THE RESIDUAL CHARACTER OF VARIETIES GENERATED BY STRICTLY SIMPLE TERM MINIMAL ALGEBRAS
}

\author{
KEITH A. KEARNES AND ÁGNES SZENDREI
}

\begin{abstract}
We prove that if $\mathbf{A}$ is a nonabelian strictly simple term minimal algebra, then the variety $\mathrm{V}(\mathbf{A})$ is either residually large or has $\mathbf{A}$ as its unique subdirectly irreducible member. We then show that it is possible to algorithmically decide the residual character of $\mathbf{V}(\mathbf{A})$ if $\mathbf{A}$ has finitely many fundamental operations.
\end{abstract}

\section{INTRODUCTION}

An algebra is called strictly simple if it is finite, simple, and has no nontrivial proper subalgebras. Strictly simple algebras are important because of the role they play in understanding the structure of locally finite minimal varieties. Every locally finite minimal variety contains a uniquely determined strictly simple algebra, and the strictly simple algebras that generate minimal varieties are characterized in [6]. Thus minimal locally finite varieties are parametrized by a recognizable family of strictly simple algebras.

Our purpose in this paper is to investigate the residual character of locally finite minimal varieties. If $\mathbf{A}$ is strictly simple and $\mathbf{V}(\mathbf{A})$ is minimal, then it is known that $\mathrm{V}(\mathbf{A})$ is residually small whenever the tame congruence theoretic type of $\mathbf{A}$ is $\mathbf{1}$ or $\mathbf{2}$. In fact, it is known that in these two cases $\mathbf{A}$ is the only subdirectly irreducible algebra in $\mathbf{V}(\mathbf{A})$. (This is proved in [2], [10], and [11].) If the tame congruence theoretic type of $\mathbf{A}$ is $\mathbf{3}$ or $\mathbf{4}$, then it is shown in [3] that $\mathrm{V}(\mathbf{A})$ is residually small if and only if it is congruence distributive. It is known that in the type $\mathbf{3}$ and $\mathbf{4}$ cases either $\mathbf{A}$ is the only subdirectly irreducible in $\mathrm{V}(\mathbf{A})$ or there is a proper class of subdirectly irreducibles.

The remaining case where the type of $\mathbf{A}$ is $\mathbf{5}$ is much harder, and is still unresolved. Examples show that $\mathbf{V}(\mathbf{A})$ can have one subdirectly irreducible, more than one but

1991 Mathematics Subject Classification. Primary 08B26, Secondary 08A05.

Key words and phrases. Residual smallness, subdirect irreducibility, strictly simple algebra, term minimal algebra, tame congruence theory.

The work of both authors was supported by the Fields Institute. The first author was supported by IRIG PCG grant no. 628318 from the University of Louisville. The second author was supported by the Hungarian National Foundation for Scientific Research grants no. T 17005 and T 022867. 
finitely many, or a proper class of them. We know of no examples where $\mathrm{V}(\mathbf{A})$ has an infinite set of subdirectly irreducible algebras, but the possibility that this can happen has not been eliminated. The fact that wild behavior occurs in type $\mathbf{5}$ is reminiscent of R. McKenzie's result in [8] that it is algorithmically undecidable whether a finite algebra of type $\mathbf{5}$ generates a residually small variety. However, McKenzie's construction involves algebras that do not generate minimal varieties. In fact, his method of construction always produces a variety with infinitely many subvarieties when his Turing machine fails to halt.

Is the residual character of a locally finite minimal variety decidable? This paper is a partial affirmative answer to this question. As we have mentioned, only the type $\mathbf{5}$ case remains unresolved. In this paper we settle the term minimal subcase for type $\mathbf{5}$ algebras. To explain how this result fits into the general question, let $\mathbf{A}$ be a strictly simple type $\mathbf{5}$ algebra. Let $e(x)$ be a nonconstant unary term operation of minimal range for which $\mathbf{A} \models e^{2}(x)=e(x)$. There is a natural way to restrict the structure of $\mathbf{A}$ to $e(A)$ which results in a 'local approximation' $e(\mathbf{A})$ of $\mathbf{A}$. The algebra $e(\mathbf{A})$ is strictly simple and term minimal, and all strictly simple term minimal algebras arise in this manner. The construction $\mathbf{A} \mapsto e(\mathbf{A})$ determines a functor $\mathbf{V}(\mathbf{A}) \rightarrow \mathbf{V}(e(\mathbf{A}))$ which preserves residual smallness. Deciding whether $\mathbf{V}(\mathbf{A})$ is residually small can be reduced to:

(i) Deciding whether $\mathbf{V}(e(\mathbf{A}))$ is residually small. (If not, then we are done: $\mathbf{V}(\mathbf{A})$ cannot be residually small.)

(ii) Deciding whether $\mathrm{V}(\mathbf{A})$ is residually small given the fact that $\mathrm{V}(e(\mathbf{A}))$ is.

We explain how to do (i) in this paper. We prove that $\mathrm{V}(e(\mathbf{A}))$ either has one subdirectly irreducible or a proper class of subdirectly irreducibles, and we give an algorithm for determining which is the case.

\section{Term Minimal Strictly Simple Algebras of Type 5}

Let $G$ be a finite group with at least two elements. We extend the multiplication of $G$ to the set $A=G \cup\{0\}$ so that 0 acts as a zero element, and we define left multiplication with an element $g \in G$ as a mapping $g \cdot: A \rightarrow A, x \mapsto g x$. Right multiplication is defined analogously. We denote by $L_{G}$ the group of left multiplications by elements of $G$. We use $R_{G}$ for the right multiplications. Clearly, $L_{G}$ and $R_{G}$ are permutation groups on $A$ which act regularly on the set $A-\{0\}=G$. We also define a binary operation $\wedge$ on $A$ by setting $a \wedge a=a$ and $a \wedge b=0$ for all $a, b \in A$ with $a \neq b$. By this definition $(A ; \wedge)$ is a height 1 semilattice with least element 0 . Moreover, it is straightforward to check that each right multiplication in $R_{G}$ is an automorphism of the algebra $\left(A ; \wedge, L_{G}, 0\right)$.

By a $G^{0}$-algebra we mean an algebra $\mathbf{A}$ with base set $A$ such that

(1) $\wedge$ and the unary operations in $L_{G} \cup\{0\}$ are term operations of $\mathbf{A}$, moreover, 
(2) the only unary term operations of $\mathbf{A}$ are the members of $L_{G} \cup\{0\}$.

Using the fact that the transformations of $A$ that commute with the operations in $L_{G} \cup\{0\}$ are those in $R_{G} \cup\{0\}$, and the fact that $\mathbf{A}$ is one-generated, one can show that the endomorphisms of $\mathbf{A}$ are exactly the members of $R_{G} \cup\{0\}$. From this it follows that in the definition of a $G^{0}$-algebra we can replace (2) with

$(2)^{\prime} R_{G} \subseteq$ Aut $\mathbf{A}$.

In particular, for every $G^{0}$-algebra $\mathbf{A}$ we have Aut $\mathbf{A}=R_{G}$.

It is easy to check that every $G^{0}$-algebra $\mathbf{A}$ is strictly simple of type $\mathbf{3}, \mathbf{4}$ or $\mathbf{5}$. Furthermore, $\mathbf{A}$ is term minimal, which means that every unary term operation $e$ satisfying $e^{2}=e$ is either constant or the identity. As the next theorem shows, the only rich class of strictly simple term minimal algebras of type $\mathbf{5}$ is the class of $G^{0}$-algebras.

Theorem 2.1. [9] The strictly simple term minimal algebras of type $\mathbf{5}$ are

(1) the algebras term equivalent to $(\mathbf{2} ; \wedge),(\mathbf{2} ; \wedge, 0),(\mathbf{2} ; \wedge, 1)$, or $(\mathbf{2} ; \wedge, 0,1)$; and

(2) the $G^{0}$-algebras of type $\mathbf{5}$.

The preceding theorem and the facts mentioned in the introduction show that the collection of $G^{0}$-algebras of type $\mathbf{5}$ is the only class of strictly simple term minimal algebras for which the residual character of the generated (minimal) variety is unknown. Therefore this paper will focus on these algebras. We will need a criterion describing when a $G^{0}$-algebra is of type $\mathbf{5}$. Such conditions were given in [9]; in Proposition 2.3 below we will recall these conditions, and will add a new one, which will be more useful for the purposes of this paper.

First, however, we need to introduce some terminology and notation. It follows from the definition of a $G^{0}$-algebra that an operation $f$ is a term operation of some $G^{0}$-algebra if and only if

$$
R_{G} \subseteq \operatorname{Aut}(A ; f) .
$$

Such an operation $f$ will be called a $G^{0}$-operation.

The natural order of the semilattice $(A ; \wedge)$ will be denoted by $\leq$, and the same symbol will be used to denote the coordinatewise order on $A^{n}$ for each $n>0$. We will say that a $G^{0}$-operation $f$ is monotone if it is monotone with respect to the semilattice order $\leq$. If $f, g$ are $G^{0}$-operations of the same arity, then $f \leq g$ will mean that $f(\bar{a}) \leq g(\bar{a})$ for every tuple $\bar{a}$ in $A$.

The unit element of the group $G$ will be denoted by 1 . As a consequence of property (2.1), every $G^{0}$-operation $f$ is uniquely determined by the set of tuples where it assumes the value 1 . This is because $f(\bar{a})=g$ for some $g \in G$ if and only if $f\left(\bar{a} g^{-1}\right)=1$, and $f(\bar{a})=0$ if and only if $f\left(\bar{a} g^{-1}\right) \neq 1$ for all $g \in G$. Thus, for any $G^{0}$-operations $f, g$ of the same arity we have 
- $f=g$ if and only if $f$ and $g$ assume the value 1 for exactly the same tuples from $A$, and

- $f \leq g$ if and only if $g$ assumes the value 1 whenever $f$ does.

Now let us consider the set of all tuples $\bar{a}$ in $A$ such that $f(\bar{a})=1$, and let $\operatorname{Min}(f)$ denote the collection of all minimal elements of this set with respect to the order $\leq$. The facts in the preceding paragraph immediately imply the following.

Lemma 2.2. For arbitrary monotone $G^{0}$-operations $f, g$ we have

(1) $f(\bar{a})=1$ if and only if $\bar{a} \geq \bar{c}$ for some $\bar{c} \in \operatorname{Min}(f)$,

(2) $f=g$ if and only if $\operatorname{Min}(f)=\operatorname{Min}(g)$, and

(3) $f \leq g$ if and only if $g(\bar{c})=1$ for all $\bar{c} \in \operatorname{Min}(f)$.

Let $\mathbf{A}$ be an arbitrary $G^{0}$-algebra. By definition, $\wedge$ and the unary operations in $L_{G} \cup\{0\}$ are term operations of $\mathbf{A}$. Therefore for every integer $k>0$, the meets of the form

$$
m(\bar{x})=c_{i_{1}}^{-1} x_{i_{1}} \wedge \cdots \wedge c_{i_{s}}^{-1} x_{i_{s}} \quad \text { with } \quad c_{i_{1}}, \ldots, c_{i_{s}} \in G, 1 \leq i_{1}<\ldots<i_{s} \leq k
$$

are $k$-ary term operations of $\mathbf{A}$. We introduce the following short notation for this operation:

$$
m(\bar{x})=\bigwedge \bar{c}^{-1} \bar{x} \quad \text { where } \quad \bar{c}=\left(c_{1}, \ldots, c_{k}\right), \quad c_{i}= \begin{cases}c_{i_{j}} & \text { if } i=i_{j}, \\ 0 & \text { otherwise }\end{cases}
$$

Observe that for a $k$-tuple $\bar{a}$ from $A^{k}$ we have $m(\bar{a})=\bigwedge \bar{c}^{-1} \bar{a}=1$ if and only if $a_{i}=c_{i}$ whenever $c_{i} \neq 0$, that is, if and only if $\bar{a} \geq \bar{c}$. Therefore the $k$-tuple $\bar{c}$ is the unique element of $\operatorname{Min}(m)$.

For arbitrary $k$-tuples $\bar{c}$ and $\bar{d}$ from $A^{k}$ we will call the $k$-ary meets $\bigwedge \bar{c}^{-1} \bar{x}$ and $\bigwedge \bar{d}^{-1} \bar{x}$ disjoint, if for every $k$-tuple $\bar{a}$ from $A^{k}$, at most one of the elements $\bigwedge \bar{c}^{-1} \bar{a}$ and $\bigwedge \bar{d}^{-1} \bar{a}$ is nonzero.

Now we are in a position to state the characterizations of type $5 G^{0}$-algebras, which we promised earlier.

Proposition 2.3. For any $G^{0}$-algebra A the following conditions are equivalent:

(i) $\mathbf{A}$ is of type $\mathbf{5}$;

(ii) no binary polynomial operation of $\mathbf{A}$ restricts as join to the set $\{0,1\}$;

(iii) the set

$$
M=\{(0,0, g, g),(0, g, 0, g),(g, g, g, g): g \in A\}
$$

is a subuniverse of $\mathbf{A}^{4}$;

(iv) every fundamental operation (hence every term operation) $f$ of $\mathbf{A}$ is monotone, and has the property that the meets $\bigwedge \bar{c}^{-1} \bar{x}$ with $\bar{c} \in \operatorname{Min}(f)$ are pairwise disjoint. 
Proof. The equivalence of (i)-(iii) was proved in [9]. Since the conditions in (i)-(iii) are invariant under term equivalence, it suffices to prove the equivalence of (iii) with the version of (iv) which refers to fundamental operations only.

Suppose first that (iii) holds. By projecting $M$ onto its first two coordinates we get the relation $\leq$, therefore every fundamental operation of $\mathbf{A}$ is monotone. Assume, in order to get a contradiction, that $\mathbf{A}$ has a fundamental operation $f$ with two distinct tuples $\bar{c}, \bar{d} \in \operatorname{Min}(f)$ such that the corresponding meets are not disjoint; say

$$
\bigwedge \bar{c}^{-1} \bar{a}=g \neq 0 \quad \text { and } \quad \bigwedge \bar{d}^{-1} \bar{a}=h \neq 0
$$

for some $\bar{a} \in A^{k}$. Using the automorphisms we see that these equalities are equivalent to $\bar{a} g^{-1} \geq \bar{c}$ and $\bar{a} h^{-1} \geq \bar{d}$. But then $f\left(\bar{a} g^{-1}\right)=1=f\left(\bar{a} h^{-1}\right)$, whence it follows that $g=f(\bar{a})=h$. Thus for the tuple $\bar{b}=\bar{a} g^{-1}$ we have $\bar{b} \geq \bar{c}, \bar{d}$, implying that $\bar{c}$ and $\bar{d}$ agree with $\bar{b}$ - and hence with each other, too - in every coordinate where both are nonzero. This shows that the list $\bar{x}$ of variables of $f$ can be partitioned into three blocks as $\left(\bar{x}_{1}, \bar{x}_{2}, \bar{x}_{3}\right)$ in such a way that for the corresponding partitions of $\bar{c}$, $\bar{d}$, and $\bar{b}$ the following conditions hold: $\bar{c}_{1}=\overline{0}, \bar{c}_{2}$ has no zero coordinate, $\bar{d}_{2}=\overline{0}$, $\bar{d}_{1}$ has no zero coordinate, and $\bar{c}_{3}=\bar{b}_{3}=\bar{d}_{3}$. Since $\bar{c}$ and $\bar{d}$ are incomparable, none of the first two blocks of this partition are empty. The minimality of $\bar{c}$ and $\bar{d}$ ensures also that $f\left(\overline{0}, \overline{0}, \bar{b}_{3}\right) \neq 1$. So by monotonicity we get the first equality below:

$$
\begin{aligned}
& f\left(\overline{0}, \overline{0}, \bar{b}_{3}\right)=0 \\
& f\left(\overline{0}, \bar{c}_{2}, \bar{b}_{3}\right)=1 \\
& f\left(\bar{d}_{1}, \overline{0}_{1}, \bar{b}_{3}\right)=1 \\
& f\left(\bar{d}_{1}, \bar{c}_{2}, \bar{b}_{3}\right)=1
\end{aligned}
$$

The second and third equalities are true because the tuples $\bar{c}=\left(\overline{0}, \bar{c}_{2}, \bar{b}_{3}\right)$ and $\bar{d}=\left(\bar{d}_{1}, \overline{0}, \bar{b}_{3}\right)$ belong to $\operatorname{Min}(f)$, and the last equality follows from the preceding two by monotonicity. Taken together, these four equalities say that computing the value of $f$ in $\mathbf{A}^{4}$ for some quadruples in $M$ we get a quadruple which fails to belong to $M$. Namely, the arguments of $f$ are quadruples of the form $(0,0, d, d),(0, c, 0, c)$, or $(b, b, b, b)$ with $b, c, d \in A$ in the first, second, or third block, respectively, and the resulting quadruple is $(0,1,1,1)$. This proves the required contradiction, and hence shows that (iii) $\Rightarrow$ (iv).

Conversely, assume that (iv) holds for $\mathbf{A}$, and consider the subuniverse $M^{\prime}$ of $\mathbf{A}^{4}$ generated by $M$. Notice that for every quadruple $\left(u_{1}, u_{2}, u_{3}, u_{4}\right) \in M$ we have $u_{1} \leq u_{l} \leq u_{4}$ for $l=2,3$, so the same will hold for all quadruples in $M^{\prime}$. Thus the elements of the difference $M^{\prime}-M$ must be of the form $(0, g, g, g)$ for some nonzero $g \in A$. Our goal is to verify that $M^{\prime}=M$. We will prove this by assuming that $M^{\prime}-M \neq \emptyset$ and deriving a contradiction.

If $M^{\prime}-M \neq \emptyset$ then $\mathbf{A}$ has a fundamental operation $f$ such that substituting appropriate quadruples from $M$ in the arguments of $f$ the resulting quadruple is of the form $(0, g, g, g)$ for some nonzero $g \in A$. Since $M$ is invariant under the 
coordinatewise action of $R_{G}$, we may assume that the arguments of $f$ are chosen so that the result is the quadruple $(0,1,1,1)$. Now let us partition the list $\bar{x}$ of variables of $f$ into three blocks as $\left(\bar{x}_{1}, \bar{x}_{2}, \bar{x}_{3}\right)$ so that $\bar{x}_{1}$ consists of those variables where the argument of $f$ is of the form $(0,0, d, d)$ for some nonzero $d \in A, \bar{x}_{2}$ consists of those variables where the argument of $f$ is of the form $(0, c, 0, c)$ for some nonzero $c \in A$, and $\bar{x}_{3}$ consists of the remaining variables where the argument of $f$ is of the form $(b, b, b, b)$ for some $b \in A$. Writing out coordinatewise what it means that the result of $f$ for these arguments is $(0,1,1,1)$ we get four equalities of the form $(2.2)$. The first three equalities imply that there exist elements $\bar{c}, \bar{d} \in \operatorname{Min}(f)$ such that

$$
\left(\overline{0}, \overline{0}, \bar{b}_{3}\right)<\bar{c} \leq\left(\overline{0}, \bar{c}_{2}, \bar{b}_{3}\right) \quad \text { and } \quad\left(\overline{0}, \overline{0}, \bar{b}_{3}\right)<\bar{d} \leq\left(\bar{d}_{1}, \overline{0}, \bar{b}_{3}\right) .
$$

Here $\bar{c}$ and $\bar{d}$ must be distinct, because

$$
\bar{c} \wedge \bar{d} \leq\left(\overline{0}, \bar{c}_{2}, \bar{b}_{3}\right) \wedge\left(\bar{d}_{1}, \overline{0}, \bar{b}_{3}\right)=\left(\overline{0}, \overline{0}, \bar{b}_{3}\right)<\bar{c}, \bar{d}
$$

Now the fourth equality in (2.2) shows that for $\bar{a}=\left(\bar{d}_{1}, \bar{c}_{2}, \bar{b}_{3}\right)$ we have $\bar{a} \geq \bar{c}$ and $\bar{a} \geq \bar{d}$. Thus $\bigwedge \bar{c}^{-1} \bar{a}=1=\bigwedge \bar{d}^{-1} \bar{a}$, which contradicts the assumption that the meets $\bigwedge \bar{c}^{-1} \bar{x}$ and $\bigwedge \bar{d}^{-1} \bar{x}$ are disjoint. This completes the proof of the implication (iv) $\Rightarrow$ (iii).

If $\bigwedge \bar{c}_{i}^{-1} \bar{x}(1 \leq i \leq m)$ is a family of pairwise disjoint $k$-ary meets, then for any $\bar{a} \in A^{k}$ at most one of the meets $\bigwedge \bar{c}_{i}^{-1} \bar{a}$ is distinct from 0 . Hence we can define a $k$-ary operation $\bigvee_{i=1}^{m}\left(\bigwedge \bar{c}_{i}^{-1} \bar{x}\right)$ on $A$ as follows: for any $\bar{a} \in A^{k}$ let

$$
\bigvee_{i=1}^{m}\left(\bigwedge \bar{c}_{i}^{-1} \bar{a}\right)=\max \left\{\bigwedge \bar{c}_{i}^{-1} \bar{a}: 1 \leq i \leq m\right\}
$$

Lemma 2.4. If $\bigwedge \bar{c}_{i}^{-1} \bar{x}(1 \leq i \leq m)$ is a family of pairwise disjoint $k$-ary meets, then the operation $\bigvee_{i=1}\left(\bigwedge \bar{c}_{i}^{-1} \bar{x}\right)$ is a monotone $G^{0}$-operation, and we have

$$
\operatorname{Min}\left(\bigvee_{i=1}^{m}\left(\bigwedge \bar{c}_{i}^{-1} \bar{x}\right)\right)=\left\{\bar{c}_{i}: 1 \leq i \leq m\right\}
$$

Proof. Let $h(\bar{x})=\bigvee_{i=1}^{m}\left(\bigwedge \bar{c}_{i}^{-1} \bar{x}\right)$. It is straightforward to check that $h$ is a $G^{0}$ operation. Furthermore, we have

$$
h(\bar{a})=1 \quad \Longleftrightarrow \quad \bar{a} \geq \bar{c}_{i} \text { for some } i .
$$

Therefore $h$ is monotone. The $k$-tuples $\bar{c}_{i}$ must be pairwise incomparable, because otherwise the joinands in $h$ would not be disjoint. Thus the property of $h$ displayed 
above implies also that $\operatorname{Min}(h)$ consists exactly of the tuples $\bar{c}_{i}(1 \leq i \leq m)$, as stated.

Proposition 2.3, combined with this lemma yields the following corollary.

Corollary 2.5. Let $\mathbf{A}$ be a $G^{0}$-algebra of type $\mathbf{5}$. Every term operation $f$ of $\mathbf{A}$ can be represented as a join of pairwise disjoint meets, and this representation is unique; explicitly, this representation is

$$
f(\bar{x})=\bigvee_{\bar{c} \in \operatorname{Min}(f)}\left(\bigwedge \bar{c}^{-1} \bar{x}\right) .
$$

For every term operation $f$ of a $G^{0}$-algebra of type $\mathbf{5}$ the join representation of $f$ described in Corollary 2.5 will be called the canonical form of $f$.

Note that a further consequence of Proposition 2.3 and Lemma 2.4 is that every $G^{0}$-algebra whose fundamental operations can be represented as joins of pairwise disjoint meets is of type 5 .

\section{Two Extremes}

Our purpose in this section is to prove the following theorem.

Theorem 3.1. For every $G^{0}$-algebra $\mathbf{A}$ of type $\mathbf{5}$ either $\mathrm{V}(\mathbf{A})$ is residually large or $\mathbf{A}$ is the only subdirectly irreducible algebra in $\mathrm{V}(\mathbf{A})$.

The argument will begin by analyzing the structure of a typical finite subdirectly irreducible algebra $\mathbf{S} \in \mathbf{V}(\mathbf{A})$. In particular, we will show that $\mathbf{S}$ has a representation where a certain 'condition $(\mathcal{R})$ ' holds. (See Lemma 3.5.) Once we have acquired enough data about $\mathbf{S}$ we will be able to show that if $\mathbf{V}(\mathbf{A})$ is residually small then 'condition $(\mathcal{R})$ ' can be strengthened to 'condition $(\mathcal{S})$ '. (See Lemma 3.7.) From condition $(\mathcal{S})$ we will be able to deduce that $\mathbf{S} \cong \mathbf{A}$.

We begin now with the first step of the project, which is to collect data about a typical subdirectly irreducible $\mathbf{S} \in \mathrm{V}(\mathbf{A})$.

Lemma 3.2. Let $\mathbf{A}$ be a $G^{0}$-algebra of type $\mathbf{5}$ and let $\mathbf{S}$ be a finite subdirectly irreducible algebra in $\mathbf{V}(\mathbf{A})$. Represent $\mathbf{S}$ as $\mathbf{B} / \delta$ for some subalgebra $\mathbf{B}$ of $\mathbf{A}^{n}$ and some congruence $\delta$ of $\mathbf{B}$. Let $\theta$ be the unique congruence of $\mathbf{B}$ which covers $\delta$, and for $1 \leq i \leq n$ let $\eta_{i}$ denote the $i$-th projection kernel on $\mathbf{B}$. Assume that none of the congruences $\bar{\eta}_{i}=\eta_{1} \cap \cdots \cap \eta_{i-1} \cap \eta_{i+1} \cap \cdots \cap \eta_{n}(1 \leq i \leq n)$ is contained in $\delta$. If $u$ is an element of $\mathbf{B}$ such that $(u, v) \in \theta-\delta$ and $v<u$ hold for some $v \in \mathbf{B}$, and $u$ is minimal with respect to this property, then

(a) $u$ has no zero coordinate, and

(b) B contains elements $c_{1}, \ldots, c_{n}$ such that $c_{i}<u$ and $\left(c_{i}, u\right) \in \bar{\eta}_{i}$ for each $1 \leq i \leq n$. 
Proof. The proof follows an argument of R. McKenzie in [7] with a slight modification. Throughout the proof $v$ will be a fixed element in $\mathbf{B}$ with the properties that $(u, v) \in$ $\theta-\delta$ and $v<u$.

Claim 3.3. For any element $b \in B$ with $b<u$ we have $(b, u) \notin \delta$.

Indeed, otherwise $b \stackrel{\delta}{\equiv} u$ implies that

$$
u \stackrel{\delta}{\equiv} b=b \wedge u \stackrel{\theta}{\equiv} b \wedge v \stackrel{\delta}{\equiv} u \wedge v=v .
$$

However, since $b<u$, the minimality property of $u$ ensures that the pair $(b, b \wedge v)$, which was shown above to belong to $\theta$, cannot lie in $\theta-\delta$. Thus $b \stackrel{\delta}{\equiv} b \wedge v$. Hence by the displayed relations we conclude that $u \stackrel{\delta}{\equiv} v$, which contradicts the choice of the pair $(u, v)$. This finishes the proof of the claim.

Claim 3.4. For any distinct elements $b, b^{\prime} \in B$ with $\left(b, b^{\prime}\right) \notin \delta$ there exist a polynomial $p \in \mathrm{Pol}_{1} \mathbf{B}$ and an element $c \in B$ such that

$$
\left\{p(b), p\left(b^{\prime}\right)\right\}=\{u, c\} \text { and } c<u \text {. }
$$

We have $\theta \subseteq \delta \vee \mathrm{Cg}^{\mathbf{B}}\left(b, b^{\prime}\right)$ and $(u, v) \in \theta$, therefore $(u, v) \in \delta \vee \mathrm{Cg}^{\mathbf{B}}\left(b, b^{\prime}\right)$. Hence there exists a sequence $u=b_{0}, b_{1}, \ldots, b_{s-1}, b_{s}=v$ in $\mathbf{B}$ such that any two consecutive elements are either $\delta$-related or are of the form $p(b), p\left(b^{\prime}\right)$ or $p\left(b^{\prime}\right), p(b)$ for some unary polynomial operation $p$ of $\mathbf{B}$. Taking $b_{i} \wedge u$ instead of $b_{i}$ throughout the sequence we may assume that $b_{i} \leq u$ for all $i$. We may also assume that $b_{1} \neq u$. Then $b_{1}<u$, so by Claim $3.3 b_{1}$ is not $\delta$-related to $u$. Hence we must have $\left\{p(b), p\left(b^{\prime}\right)\right\}=\left\{u, b_{1}\right\}$ for some $p \in \mathrm{Pol}_{1} \mathbf{B}$. The proof of the claim is complete.

Now we are in a position to prove the claims (a)-(b). Let $1 \leq i \leq n$. By assumption B contains elements $b, b^{\prime}$ such that $\left(b, b^{\prime}\right) \in \bar{\eta}_{i}-\delta$. Now we apply Claim 3.4. The element $c=c_{i}$ that we get has the properties $c_{i}<u$ and $\left(c_{i}, u\right) \in \bar{\eta}_{i}$, because $\left(b, b^{\prime}\right) \in \bar{\eta}_{i}$ implies $\left(p(b), p\left(b^{\prime}\right)\right) \in \bar{\eta}_{i}$. Thus $c_{i}$ and $u$ differ in their $i$ th coordinates only. As $c_{i}<u$, we conclude that the $i$ th coordinate of $u$ is nonzero, while the $i$ th coordinate of $c_{i}$ is zero. This implies that all coordinates of $u$ are distinct from zero.

We investigate the relationship between a $G^{0}$-algebra $\mathbf{A}$ and a typical subdirectly irreducible $\mathbf{S} \in \mathbf{V}(\mathbf{A})$ as mediated by an algebra $\mathbf{B} \leq \mathbf{A}^{n}$ in the manner described in the previous lemma. We will use the following conventions in our investigation. For $a \in A$ the constant tuple $(a, \ldots, a) \in A^{n}$ will be denoted by $\widehat{a}$. An element of $\mathbf{A}^{n}$ will be called diagonal if it is of the form $\widehat{a}$ for some $a \in A$; otherwise it will be called nondiagonal. For each $i(1 \leq i \leq n), o_{i}$ will stand for the $n$-tuple with all coordinates 1 except the $i$ th one which is 0 . A subalgebra $\mathbf{B}$ of $\mathbf{A}^{n}$ will be called diagonal if $\widehat{a} \in B$ for all $a \in A$, and rich if $\{0, a\}^{n} \subseteq B$ for all $a \in A$. Clearly, every rich subalgebra of $\mathbf{A}^{n}$ is diagonal. Because of the unary term operations in $L_{G}$, it 
follows that $\mathbf{B}$ is a diagonal subalgebra of $\mathbf{A}^{n}$ if and only if $\widehat{1} \in B$, and $\mathbf{B}$ is rich if and only if $\{0,1\}^{n} \subseteq B$. Since any tuple in $\{0,1\}^{n} \subseteq B$ which is distinct from $\widehat{1}$ is a meet of some $o_{i}$-s, therefore $\mathbf{B}$ is rich if and only if $\widehat{1}, o_{1}, o_{2}, \ldots, o_{n} \in \mathbf{B}$. If $p$ is any polynomial operation of $\mathbf{A}$ and $\mathbf{B}$ is a diagonal subalgebra of $\mathbf{A}^{n}$, then $\widehat{p}$ will stand for the polynomial operation of $\mathbf{B}$ which is $p$ acting coordinatewise. Throughout the paper we will use the symbol $e$ to denote the polynomial operation $e(x)=x \wedge 1$ of A. Hence, according to the convention just described, $\widehat{e}$ is the polynomial operation $\widehat{e}(x)=x \wedge \widehat{1}$ in each diagonal subalgebra $\mathbf{B}$ of $\mathbf{A}^{n}$.

Lemma 3.5. Let $\mathbf{A}$ be a $G^{0}$-algebra of type $\mathbf{5}$ and let $\mathbf{S} \in \mathrm{V}(\mathbf{A})$ be a finite subdirectly irreducible algebra. There exists a subalgebra $\mathbf{B}$ of $\mathbf{A}^{n}$ for some $n>0$, and a meet irreducible congruence $\delta \in$ Con $\mathbf{B}$ with unique cover $\theta$ such that

(1) $\mathbf{B}$ is rich;

(2) $\delta=\left\{\left(b, b^{\prime}\right) \in B^{2}: \widehat{e} p(b)=\widehat{1} \Longleftrightarrow \widehat{e} p\left(b^{\prime}\right)=\widehat{1} \quad\right.$ for all $\left.p \in \mathrm{Pol}_{1} \mathbf{B}\right\}$.

(3) $\mathbf{S} \cong \mathbf{B} / \delta$;

(4) $\left(o_{1}, \widehat{1}\right) \in \theta-\delta$; and

(5) the following condition holds:

$$
\widehat{e} p(\widehat{1})=\widehat{1} \quad \& \quad \widehat{e} p\left(o_{1}\right)=o_{1} \quad \Longrightarrow \quad \widehat{e} p(\widehat{0})=\widehat{0} \quad \text { for all } p \in \mathrm{Pol}_{1} \mathbf{B} .
$$

Conversely, let $\mathbf{B}$ be any rich subalgebra of $\mathbf{A}^{n}$ for which $(\mathcal{R})$ holds. If $\delta$ is defined as in $(2)$, then $\mathbf{B} / \delta$ is a finite subdirectly irreducible algebra in $\mathrm{V}(\mathbf{A})$ with monolith $\theta / \delta$ where $\theta$ is defined to be $\mathrm{Cg}^{\mathbf{B}}\left(o_{1}, \widehat{1}\right) \vee \delta$.

Proof. Let $\mathbf{S}$ be a finite subdirectly irreducible algebra in $\mathbf{V}(\mathbf{A})$. Some finite power $\mathbf{A}^{n}(n>0)$ of $\mathbf{A}$ has a subalgebra $\mathbf{B}$ which has a meet irreducible congruence $\delta$ such that (3) holds. Were one of the congruences $\bar{\eta}_{i}=\eta_{1} \cap \cdots \cap \eta_{i-1} \cap \eta_{i+1} \cap \cdots \cap \eta_{n}$ $(1 \leq i \leq n)$ contained in $\delta$, we could reduce the exponent $n$ by projecting onto $n-1$ factors. Therefore we will suppose without loss of generality that $n, \mathbf{B}$ and $\delta$ are selected so that $\bar{\eta}_{i} \nsubseteq \delta$ for all $i(1 \leq i \leq n)$.

Let $\theta$ denote the unique cover of $\delta$. Consider all pairs $(u, v) \in \theta-\delta$ such that $u>v$, and fix such a pair so that $u$ is minimal. By Lemma $3.2 u$ has no zero coordinates, and $\mathbf{B}$ contains elements $c_{1}, \ldots, c_{n}$ such that $c_{i}$ and $u$ differ in their $i$ th coordinates only, and since $c_{i}<u$, the $i$ th coordinate of $c_{i}$ is zero.

Let $u=\left(u_{1}, \ldots, u_{n}\right)$. As Aut $\mathbf{A}=R_{G}$, the following mapping is an automorphism of $\mathbf{A}^{n}$ :

$$
\mathbf{A}^{n} \rightarrow \mathbf{A}^{n}, \quad\left(a_{1}, \ldots, a_{n}\right) \mapsto\left(a_{1} u_{1}^{-1}, \ldots, a_{n} u_{n}^{-1}\right) .
$$

Replacing $\mathbf{B}, \delta, \theta$, and $u, v$ by their images under this automorphism, but retaining the same notation, we get that $u=\widehat{1}$ and $c_{i}$ is the $n$-tuple $o_{i}$ with all coordinates 1 except the $i$ th one which is 0 . Since any $n$-tuple in $\{0,1\}^{n}$ which is distinct from $\widehat{1}$ is a meet of $o_{i}$-s, it follows that $\{0,1\}^{n} \subseteq B$. Hence we have established that $\mathbf{B}$ is a rich subalgebra of $\mathbf{A}^{n}$, so (1) holds. Clearly (3) holds by construction. 
We now prove (4). We have $(\widehat{1}, v)=(u, v) \in \theta-\delta$ and $v<\widehat{1}$, therefore $v$ has at least one zero coordinate. Permuting the coordinates of $\mathbf{B}$ if necessary we may assume that the first coordinate of $v$ is zero. Then for $o_{1} \in B$ we have

$$
o_{1}=o_{1} \wedge \widehat{1} \stackrel{\theta}{\equiv} o_{1} \wedge v=v \quad \text { and } \quad o_{1}=o_{1} \wedge \widehat{1} \geq o_{1} \wedge v=v .
$$

It is impossible that $\left(o_{1}, v\right) \in \theta-\delta$ because that together with $o_{1}<\widehat{1}$ would contradict the minimality property of the element $u=\widehat{1}$. Thus $\left(o_{1}, v\right) \in \delta$, and hence $\left(o_{1}, \widehat{1}\right) \in$ $\theta-\delta$, as claimed in (4).

To show (2) notice first that $\widehat{1} / \delta=\{\widehat{1}\}$. In fact, if $b \stackrel{\delta}{\equiv} \widehat{1}$ for some $b \in B$ then $b \wedge \widehat{1} \stackrel{\delta}{\equiv} \widehat{1}$ and $b \wedge \widehat{1} \leq \widehat{1}$. Applying Claim 3.3 for $u=\widehat{1}$ we get that $b \wedge \widehat{1}=\widehat{1}$, that is, $b=\widehat{1}$.

Now let $b, b^{\prime}$ be arbitrary elements from B. Since $\{\widehat{1}\}$ is a singleton $\delta$-class, the condition

$$
\widehat{e} p(b)=\widehat{1} \quad \Longleftrightarrow \quad \widehat{e} p\left(b^{\prime}\right)=\widehat{1} \quad \text { for all } p \in \mathrm{Pol}_{1} \mathbf{B}
$$

obviously holds whenever $b \stackrel{\delta}{\equiv} b^{\prime}$. Suppose that $\left(b, b^{\prime}\right) \notin \delta$. Then by Claim 3.4 of Lemma 3.2 there exists a polynomial $p \in \mathrm{Pol}_{1} \mathbf{B}$ such that one of the elements $p(b), p\left(b^{\prime}\right)$ is $\widehat{1}$ and the other one is smaller than $\widehat{1}$. Clearly, in this case $p(b)=\widehat{e} p(b)$ and $p\left(b^{\prime}\right)=\widehat{e} p\left(b^{\prime}\right)$. So this shows that condition (3.1) fails.

To prove (5), let $p \in \mathrm{Pol}_{1} \mathbf{B}$ be such that $\widehat{e} p(\widehat{1})=\widehat{1}$ and $\widehat{e} p\left(o_{1}\right)=o_{1}$. For every $i$ $(2 \leq i \leq n)$ we have $o_{i}>o_{1} \wedge o_{i}$ and $o_{i}=\widehat{1} \wedge o_{i} \stackrel{\theta}{\equiv} o_{1} \wedge o_{i}$, so the minimality property of $\widehat{1}$ implies that $o_{i} \stackrel{\delta}{\equiv} o_{1} \wedge o_{i}$. Thus

$$
\widehat{e} p\left(o_{i}\right) \stackrel{\delta}{\equiv} \widehat{e} p\left(o_{1} \wedge o_{i}\right) \leq \widehat{e} p\left(o_{1}\right)=o_{1} .
$$

We must have $\widehat{e} p\left(o_{i}\right) \neq \widehat{1}$, since $\widehat{1} / \delta=\{\widehat{1}\}$ implies that $\widehat{1}$ is not $\delta$-related to any element $\leq o_{1}$. But $\widehat{e} p\left(o_{i}\right)(\leq \widehat{1})$ differs from $\widehat{e} p(\widehat{1})=\widehat{1}$ in its $i$ th coordinate only, therefore $\widehat{e} p\left(o_{i}\right)=o_{i}$. By assumption this equality holds for $i=1$, too. Since $\widehat{e} p(\widehat{0})$ has the same $i$ th coordinate as $\widehat{e} p\left(o_{i}\right)$, namely 0 , for every $i(1 \leq i \leq n)$, we conclude that $\widehat{e} p(\widehat{0})=\widehat{0}$, as required.

Finally, we prove that if $\mathbf{B}$ is a rich subalgebra of $\mathbf{A}^{n},(\mathcal{R})$ holds, and $\delta$ is defined as in (2), then $\mathbf{B} / \delta$ is a finite subdirectly irreducible algebra in $\mathbf{V}(\mathbf{A})$.

Claim 3.6. If $w \in B$ and $w<\widehat{1}$, then $w \stackrel{\delta}{\equiv} o_{1} \wedge w$.

Suppose that $p$ is an arbitrary unary polynomial operation of $\mathbf{B}$ such that $\widehat{e} p(w)=$ $\widehat{1}$. Since $w<\widehat{1}, w$ has a 0 coordinate. In that coordinate $\widehat{e} p(w)$ and $\widehat{e} p(\widehat{0})$ have the same value. Thus $\widehat{e} p(\widehat{0}) \neq \widehat{0}$. We have $\widehat{e} p(\widehat{1}) \geq \widehat{e} p(w)=\widehat{1}$, so $\widehat{e} p(\widehat{1})=\widehat{1}$. Hence, using the assumption $(\mathcal{R})$, we conclude that $\widehat{e} p\left(o_{1}\right) \neq o_{1}$. But $\hat{1}$ and $o_{1}$ differ in their first coordinates only and $\widehat{e p}\left(o_{1}\right) \leq \widehat{1}$, so we must have $\widehat{e p}\left(o_{1}\right)=\widehat{1}$. 
We claim that $\widehat{e} p\left(o_{1} \wedge w\right)=\widehat{1}$. Indeed, in the first coordinate we get 1 because in that coordinate $o_{1} \wedge w$ agrees with $o_{1}$, while in the remaining coordinates we get 1 because in those coordinates $o_{1} \wedge w$ agrees with $w$. This proves the implication that $\widehat{e} p(w)=\widehat{1} \Rightarrow \widehat{e} p\left(o_{1} \wedge w\right)=\widehat{1}$. The reverse implication is obvious since $o_{1} \wedge w \leq w$ and the polynomial operations of $\mathbf{B}$ are monotone. Thus $w \stackrel{\delta}{\equiv} o_{1} \wedge w$, finishing the proof of Claim 3.6.

Now we prove that $\mathbf{B} / \delta$ is subdirectly irreducible with monolith $\theta / \delta$ where $\theta=$ $\mathrm{Cg}^{\mathbf{B}}\left(o_{1}, \widehat{1}\right) \vee \delta$. By the definition of $\delta$ we have $\left(o_{1}, \widehat{1}\right) \notin \delta$, since $\widehat{e}(\widehat{1})=\widehat{1}$ but $\widehat{e}\left(o_{1}\right)=$ $o_{1} \neq \hat{1}$. Therefore it suffices to verify that $\left(o_{1}, \widehat{1}\right) \in \delta \vee \mathrm{Cg}^{\mathbf{B}}\left(b, b^{\prime}\right)$ for any pair $\left(b, b^{\prime}\right) \in B^{2}-\delta$. Choose any $\left(b, b^{\prime}\right) \in B^{2}-\delta$. By the definition of $\delta$ there exists a unary polynomial operation $p$ of $\mathbf{B}$ such that $\widehat{e} p(b)=\widehat{1}$ and $\widehat{e} p\left(b^{\prime}\right) \neq \widehat{1}$, or the same with the roles of $b, b^{\prime}$ switched. Assume the former possibility is the case, and let $w=\widehat{e} p\left(b^{\prime}\right)$. Clearly, $(\widehat{1}, w) \in \mathrm{Cg}^{\mathbf{B}}\left(b, b^{\prime}\right)$ and $w \neq \widehat{1}$. As $\widehat{1}$ is an upper bound for the elements in the range of $\widehat{e}$, we must have $w<\widehat{1}$. By Claim $3.6 o_{1} \wedge w \stackrel{\delta}{\equiv} w$, therefore

$$
o_{1}=o_{1} \wedge \widehat{1} \stackrel{\mathrm{Cg}^{\mathbf{B}}\left(b, b^{\prime}\right)}{\equiv} o_{1} \wedge w \stackrel{\delta}{\equiv} w \stackrel{\mathrm{Cg}^{\mathbf{B}}\left(b, b^{\prime}\right)}{\equiv} \widehat{1},
$$

concluding the proof.

Lemma 3.7. Let $\mathbf{A}$ be a $G^{0}$-algebra of type $\mathbf{5}$ and let $\mathbf{B}$ be a rich subalgebra of $\mathbf{A}^{n}$ for which $(\mathcal{R})$ holds. If $\mathrm{V}(\mathbf{A})$ is residually small, then $\mathbf{B}$ satisfies

$(\mathcal{S}) \quad \widehat{e} p(b)=\widehat{1} \quad \& \quad b$ nondiagonal $\Longrightarrow \widehat{e} p(\widehat{0})=\widehat{1} \quad$ for all $b \in B$ and $p \in \operatorname{Pol}_{1} \mathbf{B}$.

Proof. Assume that $\mathbf{B}$ is a rich subalgebra of $\mathbf{A}^{n}$ which satisfies $(\mathcal{R})$, but which fails to satisfy $(\mathcal{S})$. Using a construction from [4] we can show that these assumptions imply that $\mathbf{V}(\mathbf{A})$ is residually large.

Let $\eta_{1}$ denote the kernel of the first projection on $\mathbf{B} \leq \mathbf{A}^{n}$.

Claim 3.8. Let $p(x, \bar{y})$ be a polynomial of $\mathbf{B}$ and $\bar{u}, \bar{v}$ be tuples of elements of $\mathbf{B}$ which are $\eta_{1}$-related coordinatewise. If

$$
\left[\begin{array}{cc}
p(\widehat{1}, \bar{u}) & p(\widehat{1}, \bar{v}) \\
p\left(o_{1}, \bar{u}\right) & p\left(o_{1}, \bar{v}\right)
\end{array}\right]=\left[\begin{array}{ll}
\widehat{1} & s \\
r & \widehat{1}
\end{array}\right]
$$

then $r=s=\widehat{1}$.

Recall that $\bar{\eta}_{1}$ denotes the congruence on $\mathbf{B}$ which is the kernel of the projection onto all coordinates but the first. Since $\left(o_{1}, \widehat{1}\right) \in \bar{\eta}_{1}$, we deduce from the columns of the given matrix that $(\widehat{1}, r),(s, \widehat{1}) \in \bar{\eta}_{1}$. Since $\bar{u}$ and $\bar{v}$ are $\eta_{1}$-related coordinatewise, we get from the rows of the matrix that $(\widehat{1}, s),(r, \widehat{1}) \in \eta_{1}$. Thus $(\widehat{1}, r),(\widehat{1}, s) \in \eta_{1} \wedge \bar{\eta}_{1}=$ 0 .

Claim 3.9. If $\delta$ is defined as in the statement of Lemma 3.5 (2), then in $\mathbf{B}$ we have $\widehat{a} / \delta=\{\widehat{a}\}$ for every nonzero element $a \in A$. 
Because the operations in $L_{G}$ are term operations, it suffices to establish that $\widehat{1} / \delta=\{\widehat{1}\}$. If $b \in B$ is an element such that $b \stackrel{\delta}{\equiv} \widehat{1}$, then, since $\widehat{e}(\widehat{1})=\widehat{1}$, we must have $\widehat{e}(b)=\widehat{1}$, that is, $b \wedge \hat{1}=\widehat{1}$. Since $\hat{1}$ is maximal, we get $b=\hat{1}$. This completes the proof of the claim.

Claim 3.10. There is a polynomial $q(x, \bar{y})$ of $\mathbf{B}$, a pair $(a, b) \in \eta_{1}$, and tuples $\bar{c}, \bar{d}$ which are $\eta_{1}$-related coordinatewise, such that

$$
\left[\begin{array}{cc}
q(a, \bar{c}) & q(a, \bar{d}) \\
q(b, \bar{c}) & q(b, \bar{d})
\end{array}\right]=\left[\begin{array}{cc}
\widehat{1} & w \\
* & \widehat{1}
\end{array}\right],
$$

where $w<\widehat{1}$.

We show how to construct the matrix from a failure of $(\mathcal{S})$. Choose a nondiagonal $b \in B$ and a polynomial $p$ of $\mathbf{B}$ for which $\widehat{e} p(b)=\widehat{1}$ while $\widehat{e p}(\widehat{0}) \neq \widehat{1}$. Let $t$ be a term and $b_{2}, \ldots, b_{k} \in B$ be elements for which $\widehat{e} p(x)=t\left(x, b_{2}, \ldots, b_{k}\right)$. Denote the first coordinate of $b$ by $a$ and the first coordinate of every other $b_{j}$ by $a_{j}$. Since $\widehat{e} t\left(\widehat{a}, \widehat{a_{2}}, \ldots, \widehat{a_{k}}\right)$ is a diagonal element which agrees with $\widehat{e} t\left(b, b_{2}, \ldots, b_{k}\right)=\widehat{1}$ in the first coordinate, we have $\widehat{e} t\left(\widehat{a}, \widehat{a_{2}}, \ldots, \widehat{a_{k}}\right)=\widehat{1}$. Setting $w=\widehat{e} t\left(\widehat{a} \wedge b, b_{2}, \ldots, b_{k}\right)$ we get a matrix of the form:

$$
\left[\begin{array}{ll}
\widehat{e} t\left(\widehat{a} \wedge \widehat{a}, \widehat{a_{2}}, \ldots, \widehat{a_{k}}\right) & \widehat{e} t\left(\widehat{a} \wedge b, b_{2}, \ldots, b_{k}\right) \\
\widehat{e} t\left(b \wedge \widehat{a}, \widehat{a_{2}}, \ldots, \widehat{a_{k}}\right) & \widehat{e} t\left(b \wedge b, b_{2}, \ldots, b_{k}\right)
\end{array}\right]=\left[\begin{array}{cc}
\widehat{1} & w \\
* & \widehat{1}
\end{array}\right]
$$

Here each pair $(\widehat{a}, b)$ or $\left(\widehat{a}_{j}, b_{j}\right)$ is in $\eta_{1}$, and $w \leq \widehat{1}$ since $w$ is in the range of $\widehat{e}$. If $w \neq \widehat{1}$, then this matrix establishes the claim. (Here we take $q(x, \bar{y})=\widehat{e} t\left(x \wedge y_{1}, y_{2}, \ldots, y_{k}\right)$.)

We show that $w \neq \hat{1}$ must hold in the situation where $a=0$. In fact, in this case $\widehat{a}=\widehat{0}$, and so $w=\widehat{e} t\left(\widehat{a} \wedge b, b_{2}, \ldots, b_{k}\right)=\widehat{e} p(\widehat{0}) \neq \widehat{1}$. Hence the above argument always produces a suitable matrix if $a$ (the first coordinate of $b$ ) equals 0 .

Now suppose that the matrix we produced above is not of the form required by the claim; i.e., we have $\widehat{e} t\left(\widehat{a} \wedge b, b_{2}, \ldots, b_{k}\right)=w=\widehat{1}$. This forces $a \neq 0$. Furthermore, for the element $b^{\prime}=\widehat{a} \wedge b$ we have $b^{\prime}<\widehat{a}$ and $\widehat{e} p\left(b^{\prime}\right)=\widehat{1}$. Recall also that $\widehat{e} p(\widehat{0}) \neq \widehat{1}$. Therefore we can modify $p(x)$ to $p^{\prime}(x)=p(a x)$ and $b^{\prime}$ to $b^{\prime \prime}=a^{-1} b^{\prime}$, yielding:

(1) $b^{\prime \prime}<\widehat{1}$,

(2) $\widehat{e} p^{\prime}\left(b^{\prime \prime}\right)=\widehat{1}$, and

(3) $\widehat{e} p^{\prime}(\widehat{0}) \neq \widehat{1}$.

From item (1) above and Claim 3.6 of Lemma 3.5 we have $b^{\prime \prime} \stackrel{\delta}{\equiv} b^{\prime \prime} \wedge o_{1}$. Thus $\widehat{e} p^{\prime}\left(b^{\prime \prime} \wedge o_{1}\right) \stackrel{\delta}{\equiv} \widehat{e} p^{\prime}\left(b^{\prime \prime}\right)=\widehat{1}$ by item $(2)$ above. Also from Claim 3.9 we have $\widehat{1} / \delta=\{\widehat{1}\}$. Thus $\widehat{e} p^{\prime}\left(b^{\prime \prime} \wedge o_{1}\right)=\widehat{1} \neq \widehat{e} p^{\prime}(\widehat{0})$ by item (3). We must have $\widehat{0} \neq b^{\prime \prime} \wedge o_{1}<\widehat{1}$, so $b^{\prime \prime} \wedge o_{1}$ is nondiagonal. Hence we have the same starting assumptions for $b^{\prime \prime} \wedge o_{1}$ and $p^{\prime}$ that we had for $b$ and $p$. However now we have in addition that the first coordinate of $b^{\prime \prime} \wedge o_{1}$ is 0 . Therefore, as we observed in the preceding paragraph, the argument we 
gave in the first paragraph of the proof of this claim produces a matrix of the desired type. The proof of Claim 3.10 is complete.

Let $\kappa$ be any infinite cardinal. We explain now how to construct a subdirectly irreducible algebra in $\mathrm{V}(\mathbf{A})$ whose cardinality is at least $\kappa$.

Let $\mathbf{C}$ be the subalgebra of $\mathbf{B}^{\kappa}$ that consists of all tuples of the form $\left(b_{0}, b_{1}, b_{2}, \ldots\right)$ where for each $i, j<\kappa$ we have $\left(b_{i}, b_{j}\right) \in \eta_{1}$. We name some special elements of $C$ that play a role in the following argument. For each $b \in B$ we let $\widehat{b}$ denote the tuple $(b, b, b, \ldots)$, which is an element of $\mathbf{C}$. In particular, we will write $\widehat{\hat{1}}$ for $(\widehat{1}, \widehat{1}, \widehat{1}, \ldots)$. For $0<i<j<\kappa$ we define $W_{i j}$ to be the tuple whose $k$-th coordinate is the value $w$ (from Claim 3.10) if $i \leq k<j$, and which is $\widehat{1}$ otherwise:

$$
W_{i j}=(\widehat{1}, \widehat{1}, \ldots, w, w, \ldots, \underset{j}{,}, \widehat{1}, \ldots) .
$$

Note that each $W_{i j}$ is a member of $\mathbf{C}$ since $(w, \widehat{1}) \in \eta_{1}$. We define $W_{i j}^{\prime}$ to be $W_{i j} \wedge \widehat{o_{1}}$ :

$$
W_{i j}^{\prime}=\left(o_{1}, o_{1}, \ldots, w \wedge o_{i}, w \wedge \underset{j}{\left.o_{1}, \ldots, o_{1}, o_{1}, \ldots\right) .}\right.
$$

Let $\gamma$ be the congruence on $\mathbf{C}$ which is generated by all pairs of the form $\left(W_{i j}, W_{i j}^{\prime}\right)$.

Claim 3.11. $\left(\widehat{o_{1}}, \widehat{\hat{1}}\right) \notin \gamma$.

Let $E(x)=x \wedge \widehat{\widehat{1}}$. Since $\widehat{o_{1}}, \widehat{\widehat{1}} \in E(C)$, to prove the claim it suffices to show that $\left.\left(\widehat{o_{1}}, \widehat{\hat{1}}\right) \notin \gamma\right|_{E(C)}$. In fact, we will show that $\widehat{\hat{1}} /\left.\gamma\right|_{E(C)}=\{\widehat{\hat{1}}\}$. If this were not the case, then there would be a polynomial $P$ of $\mathbf{C}$ such that $E P(W)=\widehat{\widehat{1}} \neq E P\left(W^{\prime}\right)$, where $\left\{W, W^{\prime}\right\}=\left\{W_{i j}, W_{i j}^{\prime}\right\}$ for some $0<i<j<\kappa$. Since $\widehat{\hat{1}}$ is the largest element in $E(C)$ under the semilattice order, and $W_{i j}>W_{i j}^{\prime}$, it must be that $\operatorname{EP}\left(W_{i j}\right)=\widehat{\hat{1}}>\operatorname{EP}\left(W_{i j}^{\prime}\right)$. The polynomial EP may be expressed as $E t(x, \bar{U})$ where $t$ is a term and each $U_{i} \in C$. Since $E P\left(W_{i j}\right)>E P\left(W_{i j}^{\prime}\right)$, there is a coordinate $k$ where these tuples disagree. Randomly select a coordinate $\ell$ with $i \leq \ell<j$. Now we focus on the $k$-th and $\ell$-th coordinates of $E P\left(W_{i j}\right)$ and $E P\left(W_{i j}^{\prime}\right)$ : In the $k$-th coordinate we have

$$
\widehat{1}=\left(E P\left(W_{i j}\right)\right)_{k}=\widehat{e} t\left(\left(W_{i j}\right)_{k}, \bar{U}_{k}\right)=\widehat{e} t\left(\left(W_{i j}\right)_{k} \wedge \widehat{1}, \bar{U}_{k}\right)
$$

while for some $r \neq \hat{1}$ we have

$$
r=\left(E P\left(W_{i j}^{\prime}\right)\right)_{k}=\widehat{e} t\left(\left(W_{i j}^{\prime}\right)_{k}, \bar{U}_{k}\right)=\widehat{e} t\left(\left(W_{i j}\right)_{k} \wedge \widehat{o}_{1}, \bar{U}_{k}\right) .
$$

In the $\ell$-th coordinate we have

$$
\widehat{1}=\left(E P\left(W_{i j}\right)\right)_{\ell}=\widehat{e} t\left(w, \bar{U}_{\ell}\right)=\widehat{e} t\left(w \wedge \widehat{1}, \bar{U}_{\ell}\right)
$$


while

$$
\left(E P\left(W_{i j}^{\prime}\right)\right)_{\ell}=\widehat{e} t\left(w \wedge o_{1}, \bar{U}_{\ell}\right) .
$$

Since $w<\widehat{1}$ we have that $w \stackrel{\delta}{\equiv} w \wedge o_{1}$, according to Claim 3.6 of Lemma 3.5. Moreover, $\widehat{1} / \delta=\{\widehat{1}\}$, so $\widehat{1}=\widehat{e} t\left(w, \bar{U}_{\ell}\right) \stackrel{\delta}{\equiv} \widehat{e} t\left(w \wedge o_{1}, \bar{U}_{\ell}\right)$ implies that $\widehat{e} t\left(w \wedge o_{1}, \bar{U}_{\ell}\right)=\widehat{1}$. We can build a matrix from this data:

$$
\left[\begin{array}{cc}
\widehat{e} t\left(\left(W_{i j}\right)_{k} \wedge \widehat{1}, \bar{U}_{k}\right) & \widehat{e} t\left(w \wedge \widehat{1}, \bar{U}_{\ell}\right) \\
\widehat{e} t\left(\left(W_{i j}\right)_{k} \wedge \widehat{o}_{1}, \bar{U}_{k}\right) & \widehat{e} t\left(w \wedge o_{1}, \bar{U}_{\ell}\right)
\end{array}\right]=\left[\begin{array}{cc}
\widehat{1} & \widehat{1} \\
r & \widehat{1}
\end{array}\right]
$$

The assumption that $E P\left(W_{i j}\right)>E P\left(W_{i j}^{\prime}\right)$ yielded $r \neq \hat{1}$, so the existence of this matrix contradicts Claim 3.8. Thus we have $\widehat{\hat{1}} /\left.\gamma\right|_{E(C)}=\{\widehat{\hat{1}}\}$, and the claim is proved.

Claim 3.12. If $\psi \geq \gamma$ is a congruence on $\mathbf{C}$ for which $|C / \psi|<\kappa$, then $\left(\widehat{o_{1}}, \widehat{\widehat{1}}\right) \in \psi$.

Recall from Claim 3.10 that we have a polynomial $q(x, \bar{y})$ of $\mathbf{B}$, a pair $(a, b) \in \eta_{1}$, and $\eta_{1}$-related tuples $\bar{c}=\left(c_{1}, \ldots, c_{k}\right), \bar{d}=\left(d_{1}, \ldots, d_{k}\right)$ such that

$$
\left[\begin{array}{ll}
q(a, \bar{c}) & q(a, \bar{d}) \\
q(b, \bar{c}) & q(b, \bar{d})
\end{array}\right]=\left[\begin{array}{cc}
\widehat{1} & w \\
* & \widehat{1}
\end{array}\right] .
$$

For each $i<\kappa$ and $1 \leq j \leq k$ the algebra $\mathbf{C}$ contains the elements

$$
a^{i}=(a, a, a, \ldots, b, b, b, \ldots) \quad \text { and } \quad c_{j}^{i}=\left(c_{j}, c_{j}, c_{j}, \ldots, d_{j}, d_{j}, d_{j}, \ldots\right) .
$$

Moreover, the polynomial $\widehat{q}(x, \bar{y})$, which is $q$ acting coordinatewise, is a polynomial of $\mathbf{C}$. If $\psi$ is a congruence on $\mathbf{C}$ for which $|C / \psi|<\kappa$, then there exist $0<i<j<\kappa$ such that $a^{i} \stackrel{\psi}{\equiv} a^{j}$. Therefore $\widehat{\widehat{1}}=\widehat{q}\left(a^{i}, c_{1}^{i}, c_{2}^{i}, \ldots, c_{k}^{i}\right) \stackrel{\psi}{\equiv} \widehat{q}\left(a^{j}, c_{1}^{i}, c_{2}^{i}, \ldots, c_{k}^{i}\right)=W_{i j}$. This proves that $\left(\widehat{\hat{1}}, W_{i j}\right) \in \psi$ for some $0<i<j<\kappa$ whenever $\psi$ has index $<\kappa$. But when $\left(\widehat{\hat{1}}, W_{i j}\right) \in \psi$, then we also have

$$
\left(\widehat{o_{1}}, W_{i j}^{\prime}\right)=\left(\widehat{\hat{1}} \wedge \widehat{o_{1}}, W_{i j} \wedge \widehat{o_{1}}\right) \in \psi,
$$

and consequently $\widehat{\widehat{1}} \stackrel{\psi}{\equiv} W_{i j} \stackrel{\gamma}{=} W_{i j}^{\prime} \stackrel{\psi}{\equiv} \widehat{o_{1}}$. Thus if $\psi \geq \gamma$ and $|C / \psi|<\kappa$, then $\left(\widehat{\hat{1}}, \widehat{o_{1}}\right) \in \psi$.

To finish the proof, recall that by Claim 3.11 we have $\left(\widehat{o_{1}}, \widehat{\widehat{1}}\right) \notin \gamma$. If we extend $\gamma$ to a congruence $\psi$ which is maximal for $\left(\widehat{o_{1}}, \widehat{\hat{1}}\right) \notin \psi$, then the quotient $\mathbf{C} / \psi$ will be subdirectly irreducible by construction and it will have cardinality at least $\kappa$ by Claim 3.12. Thus $\mathbf{V}(\mathbf{A})$ has a subdirectly irreducible algebra of cardinality $\geq \kappa$ for every $\kappa$. This finishes the proof.

Lemma 3.13. Let $\mathbf{A}$ be a $G^{0}$-algebra of type $\mathbf{5}$. The following conditions are equivalent. 
(i) $\mathrm{V}(\mathbf{A})$ is residually small.

(ii) Every rich subalgebra $\mathbf{B}$ of $\mathbf{A}^{n}$ which satisfies $(\mathcal{R})$ also satisfies $(\mathcal{S})$.

(iii) Up to isomorphism, $\mathbf{A}$ is the only subdirectly irreducible algebra in $\mathrm{V}(\mathbf{A})$.

Proof. The implication (iii) $\Rightarrow(\mathrm{i})$ is trivial, and the implication (i) $\Rightarrow($ ii) is proved in Lemma 3.7. Therefore we only have to prove that (ii) $\Rightarrow$ (iii).

Assume that (ii) holds. To prove that $\mathbf{A}$ is the only subdirectly irreducible algebra in $\mathbf{V}(\mathbf{A})$ it suffices to prove that there are no other finite subdirectly irreducible algebras in $\mathbf{V}(\mathbf{A})$. If $\mathbf{S}$ is any finite subdirectly irreducible algebra in $\mathbf{V}(\mathbf{A})$, then according to Lemma 3.5 we can represent $\mathbf{S}$ as $\mathbf{B} / \delta$ for some rich subalgebra $\mathbf{B}$ of $\mathbf{A}^{n}$ in such a way that $(\mathcal{R})$ holds and $\delta$ is defined as in Lemma 3.5. By (ii) we have that $(\mathcal{S})$ holds for $\mathbf{B}$.

By Claim 3.9 of Lemma 3.7, which holds for any diagonal subalgebra $\mathbf{B}$ of $\mathbf{A}^{n}$, to prove that $\mathbf{B} / \delta \cong \mathbf{A}$ it suffices to show that every nondiagonal $b \in B$ is $\delta$-related to $\widehat{0}$. Choose a nondiagonal $b \in B$. By $(\mathcal{S})$, for any unary polynomial $p$ we have $\widehat{e} p(b)=\widehat{1} \Longrightarrow \widehat{e} p(\widehat{0})=\widehat{1}$. Conversely, $\widehat{e} p(\widehat{0})=\widehat{1} \Longrightarrow \widehat{e} p(b)=\widehat{1}$ by the monotonicity of the operations of $\mathbf{B}$. Hence $b \stackrel{\delta}{\equiv} \widehat{0}$ for any nondiagonal $b$, and we are done.

Theorem 3.1 is a direct corollary of Lemma 3.13.

The result that we have just proved, that the variety $\mathrm{V}(\mathbf{A})$ is residually small if and only if $\mathbf{A}$ is the only subdirectly irreducible in $\mathbf{V}(\mathbf{A})$, depends essentially on the assumption that $\mathbf{A}$ is term minimal. To see this, we describe now an example of a strictly simple algebra $\mathbf{A}$ of type $\mathbf{5}$ which generates a residually small minimal variety with more than one subdirectly irreducible member.

Example 3.14. Our algebra will be an expansion of the three-element linearlyordered meet semilattice $(\{0,1,2\} ; \wedge)$ with $0<1<2$. To this we add binary operation $*$ defined by

$$
x * y= \begin{cases}x & \text { if } x, y \in\{1,2\} \\ 0 & \text { otherwise. }\end{cases}
$$

Finally we add unary operations $s$ and $t$ defined by $s(0)=0, s(1)=s(2)=2$ and $t(0)=t(1)=0$ and $t(2)=1$. The algebra is $\mathbf{A}=(\{0,1,2\} ; \wedge, *, s, t)$. The following claims about $\mathbf{A}$ can be easily verified by hand:

(1) $\mathbf{A}$ is strictly simple.

(2) The semilattice operation on $\mathbf{A}$ commutes with all other operations.

(3) $s$ is an idempotent unary term of $\mathbf{A}$ which has minimal range, and $\mathbf{A}$ satisfies

$$
x=x * s(x), \quad x * s t(x)=s(x) * s t(x), \quad s(x) * s(x)=s(x) .
$$

Properties (1) and (2) imply that $\mathbf{A}$ is of type $\mathbf{5}$. Property (2) implies that $\mathbf{V}(\mathbf{A})$ has a finite bound on the sizes of its subdirectly irreducible members, by Theorem 5.1 of 
[5]. Property (3) implies that $\mathbf{V}(\mathbf{A})$ is a minimal variety, according to Theorem 3.3 of $[6]$.

What remains to show is that $\mathrm{V}(\mathbf{A})$ contains a subdirectly irreducible algebra that is not isomorphic to A. A four-element subdirectly irreducible can be constructed in $\mathbf{V}(\mathbf{A})$ by these steps: Let $\mathbf{B}$ be the subalgebra of $\mathbf{A}^{2}$ that consists of all $(a, b)$ with $a \leq b$ in the semilattice order. Let $\delta$ denote the congruence on $\mathbf{B}$ generated by $\langle(0,0),(0,2)\rangle$. Then $\delta$ partitions $B$ into four blocks:

$$
(0,0)(0,1)(0,2) /(1,1) /(1,2) /(2,2) .
$$

The quotient algebra is subdirectly irreducible with monolith $\langle(1,1) / \delta,(1,2) / \delta\rangle$.

\section{Characterization}

The theorem in [4] which characterizes those finite algebras $\mathbf{A}$ for which there is a bound on the sizes of the subdirectly irreducible algebras with nonabelian monoliths in $\mathrm{V}(\mathbf{A})$ involves all finite subdirectly irreducible algebras in $\mathrm{V}(\mathbf{A})$. In fact, as it was shown by R. McKenzie in [8], there is no algorithm which, for a finite algebra A with finitely many fundamental operations, decides whether the variety $\mathrm{V}(\mathbf{A})$ is residually small. We will find in this section that $G^{0}$-algebras of type $\mathbf{5}$ are much more tractable: among $G^{0}$-algebras of type 5 those generating residually small varieties are characterized by a condition on $(k+1)$-ary term operations for each $k$-ary fundamental operation of $\mathbf{A}$. Thus it will follow that for algebras with finitely many fundamental operations, there is an algorithm which decides whether the generated variety is residually small.

If $f(\bar{x})$ is a $k$-ary term operation of a $G^{0}$-algebra of type $\mathbf{5}$ and the canonical form of $f$ is

$$
f(\bar{x})=\bigvee_{i=1}^{m}\left(\bigwedge \bar{c}_{i}^{-1} \bar{x}\right) \quad \text { for some } k \text {-tuples } \bar{c}_{i}(1 \leq i \leq m),
$$

then $\tilde{f}$ will denote the $(k+m)$-ary operation

$$
\widetilde{f}(\bar{x}, \bar{z})=\widetilde{f}\left(x_{1}, \ldots, x_{k}, z_{1}, \ldots, z_{m}\right)=\bigvee_{i=1}^{m}\left(z_{i} \wedge \wedge \bar{c}_{i}^{-1} \bar{x}\right),
$$

and for each $j(1 \leq j \leq m), \widetilde{f}^{(j)}$ will denote the $(k+1)$-ary operation

$$
\widetilde{f}^{(j)}(\bar{x}, z)=\widetilde{f}^{(j)}\left(x_{1}, \ldots, x_{k}, z\right)=\left(z \wedge \bigwedge \bar{c}_{j}^{-1} \bar{x}\right) \vee \bigvee_{\substack{i=1 \\ i \neq j}}^{m}\left(\bigwedge \bar{c}_{i}^{-1} \bar{x}\right)
$$


Since the joinands in the canonical form of $f$ are pairwise disjoint, it is easy to see that the joinands in the definition of $\widetilde{f}$ and $\widetilde{f}^{(j)}(1 \leq j \leq m)$ are also such. Thus these operations are given in canonical form.

Notice that the definition of $\widetilde{f}$ and $\widetilde{f}^{(j)}(1 \leq j \leq m)$ require a fixed indexing of the joinands of the canonical form of $f$. Therefore whenever we will mention any of the operations $\widetilde{f}$ and $\widetilde{f}^{(j)}$, we will assume that this indexing is fixed in advance. A change in the indexing of the joinands of $f$ results in a permutation of the variables $\bar{z}$ in $\widetilde{f}$, and a change in the indexing of the family $\left\{\widetilde{f}^{(j)}: 1 \leq j \leq m\right\}$. None of these will affect the statements of the results.

Theorem 4.1. Let $\mathbf{A}$ be a $G^{0}$-algebra of type $\mathbf{5}$. The following conditions are equivalent:

(i) $\mathrm{V}(\mathbf{A})$ is residually small (or equivalently, $\mathbf{A}$ is the only subdirectly irreducible algebra in $\mathrm{V}(\mathbf{A})$ ).

(ii) For every fundamental operation $f$ of $\mathbf{A}, \tilde{f}$ is a term operation of $\mathbf{A}$.

(iii) For every fundamental operation $f$ of $\mathbf{A}$, all $\widetilde{f}^{(j)}$ are term operations of $\mathbf{A}$.

Observe that condition (i) of the theorem is invariant under term equivalence, so conditions (ii) and (iii) could be replaced by the same requirements for all term operations in place of all fundamental operations. The equivalence of (ii) to its variant for term operations, and the analogous statement for (iii), can also be proved in a straightforward manner, using induction on the lengths of terms.

It is clear that for each operation $f$ of a $G^{0}$-algebra of type 5 the canonical form of $f$ is effectively computable, and hence so is the family of operations $\widetilde{f}^{(j)}$. Thus there is an algorithm which decides whether condition (iii) holds, provided the algebra has only finitely many fundamental operations. Thus Theorem 4.1 yields the following corollary.

Corollary 4.2. There is an algorithm which, for every $G^{0}$-algebra $\mathbf{A}$ of type $\mathbf{5}$ with finitely many fundamental operations, decides whether the variety $\mathrm{V}(\mathbf{A})$ is residually small.

This corollary can also be derived from our Lemma 3.13 and Theorem 5.1 of [12].

The rest of this section is devoted to the proof of Theorem 4.1. The easy equivalence of conditions (ii) and (iii) is verified in Lemma 4.3, while the implications (ii) $\Rightarrow$ (i) and (i) $\Rightarrow$ (iii) are the content of Lemmas 4.5 and 4.7 , respectively.

Lemma 4.3. Let $f$ be a term operation of a $G^{0}$-algebra of type $\mathbf{5}$, and assume that $f$ has canonical form (4.1). Then the $G^{0}$-algebras

$$
\left(A ; \wedge, L_{G}, 0, \widetilde{f}\right) \quad \text { and } \quad\left(A ; \wedge, L_{G}, 0,\left\{\widetilde{f}^{(j)}: 1 \leq j \leq m\right\}\right)
$$

are term equivalent. 
Proof. It is straightforward to check that

$$
\widetilde{f}\left(\bar{x}, z_{1}, \ldots, z_{m}\right)=\bigwedge_{j=1}^{m} \widetilde{f}^{(j)}\left(\bar{x}, z_{j}\right),
$$

and

$$
\widetilde{f}^{(j)}(\bar{x}, z)=\widetilde{f}\left(\bar{x}, \bigwedge \bar{c}_{1}^{-1} \bar{x}, \ldots, \bigwedge \bar{c}_{j-1}^{-1} \bar{x}, z, \bigwedge \bar{c}_{j+1}^{-1} \bar{x}, \ldots, \bigwedge \bar{c}_{m}^{-1} \bar{x}\right)
$$

for every $j(1 \leq j \leq m)$.

Now we start the proof that in Theorem 4.1 condition (ii) implies condition (i). Our approach will be based on the result of Lemma 3.13: we will show that if condition (ii) holds and $\mathbf{B}$ is a rich subalgebra of $\mathbf{A}^{n}$ which fails to satisfy $(\mathcal{S})$, then $\mathbf{B}$ fails to satisfy $(\mathcal{R})$. First we analyze what it means for $\mathbf{B}$ to fail $(\mathcal{S})$.

A failure of condition $(\mathcal{S})$ for $\mathbf{B}$ means that for some $k>0$ there exist a $k$-ary term $t$ and elements $b_{1}, b_{2}, \ldots, b_{k} \in B$ such that $b_{1}$ is nondiagonal and

$$
\widehat{e} t\left(b_{1}, b_{2}, \ldots, b_{k}\right)=\widehat{1}, \quad \text { but } \widehat{e} t\left(\widehat{0}, b_{2}, \ldots, b_{k}\right) \neq \widehat{1} \text {. }
$$

Since $t$ is monotone and for an element $b \in B$ we have $\widehat{e}(b)=\widehat{1}$ if and only if $b=\widehat{1}$, the displayed properties are equivalent to requiring that

$$
t\left(b_{1}, b_{2}, \ldots, b_{k}\right)=\widehat{1}, \quad \text { but } t\left(\widehat{0}, b_{2}, \ldots, b_{k}\right)<\widehat{1} .
$$

If for a term $t$ there exist elements $b_{1}, b_{2}, \ldots, b_{k} \in B$ such that $b_{1}$ is nondiagonal and (4.2) holds, then we will say that $t$ witnesses the failure of condition $(\mathcal{S})$.

The next lemma shows that if condition $(\mathcal{S})$ fails for some $\mathbf{B}$, then this failure is witnessed by a term operation which is almost a fundamental operation.

Lemma 4.4. Let $\mathbf{A}$ be a $G^{0}$-algebra of type $\mathbf{5}$, and let $\mathbf{B}$ be a rich subalgebra of $\mathbf{A}^{n}$. If condition $(\mathcal{S})$ fails for $\mathbf{B}$, then this failure is witnessed by a term operation of the form $t\left(x_{1}, \ldots, x_{k}\right)=$ af $\left(x_{1 \pi}, \ldots, x_{k \pi}\right)$ where $a \in G, f$ is a fundamental operation, and $\pi$ is a permutation of the set $\{1, \ldots, k\}$.

Proof. Let us fix a term $t$ witnessing the failure of condition $(\mathcal{S})$, and select $t$ so that $t$ has the least possible number of nonunary operation symbols. Let $b_{1}, b_{2}, \ldots, b_{k} \in B$ be elements from $\mathbf{B}$ such that $b_{1}$ is nondiagonal and (4.2) holds. It is not the case that $t$ is essentially unary, because then $t$ has to depend on its first variable, and a unary term operation cannot produce $\widehat{1}$ from a nondiagonal element $b_{1}$. Thus $t$ is of the form

$$
t(\bar{x})=a f\left(t_{1}(\bar{x}), t_{2}(\bar{x}), \ldots, t_{m}(\bar{x})\right)
$$

for some $a \in G$, some fundamental operation $f$ which is $m$-ary $(m \geq 2)$, and some terms $t_{l}(1 \leq l \leq m)$. Let $c_{l}=t_{l}\left(b_{1}, b_{2}, \ldots, b_{k}\right)(1 \leq l \leq m)$. By permuting the variables of $f$ we may assume that the elements $c_{1}, \ldots, c_{s}$ are nondiagonal, while $c_{s+1}, \ldots, c_{m}$ are diagonal $(0 \leq s \leq m)$. 
Now let $s+1 \leq l \leq m$. Then $c_{l}=\widehat{a_{l}}$ for some $a_{l} \in A$, and the term $a_{l}^{-1} t_{l}$ has the property $a_{l}^{-1} t_{l}\left(b_{1}, b_{2}, \ldots, b_{k}\right)=\widehat{1}$ where $b_{1}$ is nondiagonal. By the choice of $t$ the term $t_{l}$ cannot witness the failure of $(\mathcal{S})$, therefore $a_{l}^{-1} t_{l}\left(\widehat{0}, b_{2}, \ldots, b_{k}\right)=\widehat{1}$, that is,

$$
t_{l}\left(\widehat{0}, b_{2}, \ldots, b_{k}\right)=\widehat{a}_{l}=c_{l} .
$$

Thus

$$
\begin{aligned}
a f\left(\widehat{0}, \ldots, \widehat{0}, c_{s+1}, \ldots, c_{m}\right) & \leq a f\left(t_{1}\left(\widehat{0}, b_{2}, \ldots, b_{k}\right), \ldots, t_{s}\left(\widehat{0}, b_{2}, \ldots, b_{k}\right), c_{s+1}, \ldots, c_{m}\right) \\
& =a f\left(t_{1}\left(\widehat{0}, b_{2}, \ldots, b_{k}\right), \ldots, t_{m}\left(\widehat{0}, b_{2}, \ldots, b_{k}\right)\right) \\
& =t\left(\widehat{0}, b_{2}, \ldots, b_{k}\right) \\
& <\widehat{1}
\end{aligned}
$$

and

$$
a f\left(c_{1}, \ldots, c_{s}, c_{s+1}, \ldots, c_{m}\right)=\widehat{1}
$$

So the sequence

$$
\begin{aligned}
a f\left(\widehat{0}, \ldots, \widehat{0}, c_{s+1}, \ldots, c_{m}\right) & \leq a f\left(\widehat{0}, \ldots, \widehat{0}, c_{s}, \ldots, c_{m}\right) \leq \ldots \\
& \leq a f\left(\widehat{0}, c_{2}, \ldots, c_{m}\right) \leq a f\left(c_{1}, \ldots, c_{m}\right)=\widehat{1}
\end{aligned}
$$

contains two consecutive members such that the first one is less than $\hat{1}$ and the second one is equal to $\widehat{1}$. With the elements appearing there an operation arising from af by permuting variables witnesses the failure of $(\mathcal{S})$.

Lemma 4.5. If $\mathbf{A}$ is a $G^{0}$-algebra of type $\mathbf{5}$ such that for every fundamental operation $f$ of $\mathbf{A}, \widetilde{f}$ is a term operation of $\mathbf{A}$, then $\mathbf{V}(\mathbf{A})$ is residually small.

Proof. Assume that A satisfies the hypotheses of the lemma, and consider a rich subalgebra $\mathbf{B}$ of $\mathbf{A}^{n}(n>0)$ for which condition $(\mathcal{S})$ fails. Lemma 3.13 says that we are done if we are able to show that condition $(\mathcal{R})$ must fail for $\mathbf{B}$.

By Lemma 4.4 there exist a fundamental operation $f$ and an element $a \in G$ such that a term operation $t$ arising from af by permuting its variables witnesses the failure of $(\mathcal{S})$. By assumption, $\widetilde{f}$ is a term operation of $\mathbf{A}$. It is easy to show that $\widetilde{t}$, too, is a term operation of $\mathbf{A}$. This follows by observing that if $g$ arises from $f$ by permuting its variables then $\widetilde{g}$ arises the same way from $\widetilde{f}$; furthermore, if $g=a f$ for some $a \in G$ then

$$
\widetilde{g}(\bar{x}, \bar{z})=a \widetilde{f}\left(\bar{x}, a^{-1} \bar{z}\right)
$$

Using Lemma 4.3 we conclude that all $\widetilde{t}^{(j)}$ as well, are term operations of $\mathbf{A}$.

Let $t$ be $k$-ary and let $t$ have canonical form

$$
t(\bar{x})=\bigvee_{i=1}^{m}\left(\bigwedge \bar{c}_{i}^{-1} \bar{x}\right) \quad \text { for some } k \text {-tuples } \bar{c}_{i}(1 \leq i \leq m) .
$$

Further, let $b_{j}=\left(b_{1 j}, \ldots, b_{n j}\right)(1 \leq j \leq k)$ be elements from $\mathbf{B}$ such that $b_{1}$ is nondiagonal and (4.2) holds. Let us form the $n \times k$ matrix whose columns are the 
$n$-tuples $b_{1}, \ldots, b_{k}$, and denote the rows of this matrix by $\bar{r}_{1}, \ldots, \bar{r}_{n}$. The equality $t\left(b_{1}, \ldots, b_{k}\right)=\widehat{1}$ means that $t\left(\bar{r}_{i}\right)=1$ for each $i(1 \leq i \leq n)$. Hence, for each $i$ $(1 \leq i \leq n)$, there exists an index $s_{i}\left(1 \leq s_{i} \leq m\right)$ such that $\bar{r}_{i} \geq \bar{c}_{s_{i}}$. We may assume without loss of generality that the joinands of $t$ are indexed so that $\bar{r}_{1} \geq \bar{c}_{1}$. Let $\bar{c}_{1}=\left(c_{11}, \ldots, c_{1 k}\right)$.

We claim that $\bar{r}_{l} \nsupseteq \bar{c}_{1}$ for at least one $l(1 \leq l \leq n)$. Suppose otherwise, that is, $\bar{r}_{i} \geq \bar{c}_{1}$ for all $i(1 \leq i \leq n)$. Then $b_{i j}=c_{1 j}$ whenever $c_{1 j} \neq 0$. This means that $b_{j}=\widehat{c_{1 j}}$ is diagonal whenever $c_{1 j} \neq 0$. Since $b_{1}$ is nondiagonal, we have $c_{11}=0$, and hence

$$
\widehat{1}=c_{11}^{-1} \widehat{0} \wedge c_{12}^{-1} b_{2} \wedge \cdots \wedge c_{1 k}^{-1} b_{k} \leq t\left(\widehat{0}, b_{2}, \ldots, b_{k}\right)<\widehat{1}
$$

which is impossible.

Let us fix an index $l$ such that $\bar{r}_{l} \nsupseteq \bar{c}_{1}$. Then

$$
\widetilde{t}^{(1)}\left(\bar{r}_{l}, 0\right)=1 \quad \text { and } \quad \widetilde{t}^{(1)}\left(\bar{r}_{1}, 0\right)=0 .
$$

Furthermore, it is clear that

$$
\widetilde{t}^{(1)}\left(\bar{r}_{i}, 1\right)=1 \quad \text { for all } i(1 \leq i \leq n) .
$$

Now consider the unary polynomial operation $p(z)=\widetilde{t}^{(1)}\left(b_{1}, b_{2}, \ldots, b_{k}, z\right)$ of $\mathbf{B}$. Computing the values coordinatewise one can easily check that $\widehat{e} p(\widehat{1})=\widehat{1}, \widehat{e} p\left(o_{1}\right)=o_{1}$, but $\widehat{e} p(\widehat{0})>\widehat{0}$ as the $l$ th coordinate of $\widehat{e} p(\widehat{0})$ is 1 . Thus condition $(\mathcal{R})$ fails for $\mathbf{B}$.

Lemma 4.6. Let $\mathbf{A}$ be a $G^{0}$-algebra of type $\mathbf{5}$, and suppose that the variety $\mathrm{V}(\mathbf{A})$ is residually small. If $f$ is a $k$-ary term operation of $\mathbf{A}$ with canonical form (4.1), then A has a $(k+1)$-ary term operation $t$ which satisfies the following equalities:

$$
\begin{aligned}
& t\left(\bar{c}_{1}, 1\right)=1 \\
& t\left(\bar{c}_{1}, 0\right)=0 \\
& t\left(\bar{c}_{i}, 0\right)=1 \quad \text { for } i=2, \ldots, m .
\end{aligned}
$$

Proof. Consider a $k$-ary term operation $f$ of $\mathbf{A}$ with canonical form (4.1), and let $I$ be any subset of $\{2, \ldots, m\}$. We will show by induction on $|I|$ that $\mathbf{A}$ has a $(k+1)$-ary term operation $t$ satisfying the three equalities displayed in the lemma for $i \in I$. Clearly, the case $I=\{2, \ldots, m\}$ yields the required term operation.

For $I=\emptyset$ the claim is obvious: we can take the term operation $t(\bar{x}, z)=z$. Now assume that $I$ is nonempty and that an appropriate term operation exists for all proper subsets of $I$. For simplicity of notation assume that $I=\{2, \ldots, n\}(n \leq m)$, and consider the columns $b_{j}=\left(c_{1 j}, \ldots, c_{n j}\right)(1 \leq j \leq k)$ of the $n \times k$ matrix whose rows are the first $n$ tuples $\bar{c}_{i}=\left(c_{i 1}, \ldots, c_{i k}\right)(1 \leq i \leq n)$ from the canonical form of $f$. Recall that $o_{i}$ denotes the $n$-tuple with all coordinates 1 but the $i$-th one which is $0(1 \leq i \leq n)$, and let $\mathbf{B}$ be the subalgebra of $\mathbf{A}^{n}$ generated by the set

$$
\left\{b_{1}, \ldots, b_{k}\right\} \cup\left\{\widehat{1}, o_{1}, \ldots, o_{n}\right\} .
$$


In other words, $\mathbf{B}$ is the smallest rich subalgebra of $\mathbf{A}^{n}$ which contains the elements $b_{1}, \ldots, b_{k}$.

First we prove that $f$ (more precisely, an operation arising from $f$ by permuting its variables) witnesses the failure of $(\mathcal{S})$ in B. Clearly, we have $f\left(b_{1}, \ldots, b_{k}\right)=\widehat{1}$. Since $n \geq 2$ and the rows $\bar{c}_{1}, \ldots, \bar{c}_{n}$ are pairwise distinct, at least one of the columns $b_{1}, \ldots, b_{k}$ is nondiagonal. Assume without loss of generality that $b_{1}$ is nondiagonal; in particular, $b_{1} \neq \widehat{0}$. Let, say, $c_{i 1} \neq 0$. Since $\bar{c}_{i}$ is a minimal element for which $f$ assumes the value 1 , by changing its nonzero first coordinate to zero yields a tuple where $f$ assumes the value 0 . Therefore $f\left(\widehat{0}, b_{2}, \ldots, b_{k}\right)<\widehat{1}$, as the $i$ th coordinate of the left hand side is 0 .

The preceding paragraph shows that condition $(\mathcal{S})$ fails for B. However, by assumption, the variety $\mathrm{V}(\mathbf{A})$ is residually small. Hence by Lemma 3.13 condition $(\mathcal{R})$ has to fail for $\mathbf{B}$. That is, there exists a unary polynomial operation $p$ of $\mathbf{B}$ such that

$$
\widehat{e} p(\widehat{1})=\widehat{1}, \quad \widehat{e} p\left(o_{1}\right)=o_{1}, \text { but } \widehat{e} p(\widehat{0})>\widehat{0} .
$$

We can write the polynomial operation $\widehat{e} p$ as

$$
\widehat{e p}(z)=t^{\prime}\left(b_{1}, \ldots, b_{k}, \widehat{1}, o_{1}, o_{2}, \ldots, o_{n}, z\right)
$$

for some term $t^{\prime}$ where the parameters are from the generating set of $\mathbf{B}$. Now replace the parameters $o_{2}, \ldots, o_{n}$ by $\widehat{1}$. By monotonicity the values at $\widehat{1}, o_{1}$, and $\widehat{0}$ can only increase, but have to remain within the set $\widehat{e}(B)=\{0,1\}^{n}$. Thus the value at $\widehat{1}$ will remain $\widehat{1}$, the value at $\widehat{0}$ will continue to be greater than $\widehat{0}$, and the value at $o_{1}$ will be $o_{1}$ or $\widehat{1}$. However, since the change we made does not affect the first coordinate, we get that the value at $o_{1}$ will be $o_{1}$. We can apply the same trick for $o_{1}$, too, provided that we are able to prove that the value at $o_{1}$ will remain $o_{1}$. Suppose not, that is, changing the parameter $o_{1}$ to $\widehat{1}$ changes the value at $o_{1}$ from $o_{1}$ to $\widehat{1}$. Then let's look at the first coordinates of all equalities we know, and compute some values of the polynomial operation

$$
p_{1}(x, y)=t^{\prime}\left(\bar{c}_{1}, 1, x, 1, \ldots, 1, y\right)
$$

of A. From the original equalities (where $o_{1}$ is unchanged), by putting $z=\hat{1}$ and $z=o_{1}$, respectively, we get that $p_{1}(0,1)=1$ and $p_{1}(0,0)=0$. From the assumption that changing the parameter $o_{1}$ to $\widehat{1}$ changes the value at $o_{1}$ from $o_{1}$ to $\widehat{1}$, we get that $p_{1}(1,0)=1$. By monotonicity these equalities show that $p_{1}$ restricts to the set $\{0,1\}$ as join, which is impossible (cf. Proposition 2.3). This proves that all parameters $o_{1}, o_{2}, \ldots, o_{n}$ can be changed to $\hat{1}$ so that the required properties of $p$ in (4.3) remain valid. Finally, each occurrence of $\widehat{1}$ as a parameter in the polynomial can be replaced by $f\left(b_{1}, \ldots, b_{k}\right)$. 
These arguments show that we can always select a polynomial $p$ satisfying (4.3) where the only parameters that occur in the expression of $\widehat{e} p(z)$ are $b_{1}, \ldots, b_{k}$. Accordingly, from now on, let

$$
\widehat{e} p(z)=t^{\prime}\left(b_{1}, \ldots, b_{k}, z\right)
$$

for some term $t^{\prime}$. Now the equations (4.3), viewed coordinatewise, mean that

$$
\begin{aligned}
t^{\prime}\left(\bar{c}_{i}, 1\right) & =1 \quad \text { for all } i(1 \leq i \leq n), \\
t^{\prime}\left(\bar{c}_{1}, 0\right) & =0, \\
t^{\prime}\left(\bar{c}_{l}, 0\right) & =1 \quad \text { for some } l(2 \leq l \leq n) .
\end{aligned}
$$

Applying the induction hypothesis for the proper subset $I-\{l\}$ of $I=\{2, \ldots, n\}$ we know that $\mathbf{A}$ has a $(k+1)$-ary term operation $t^{\prime \prime}$ such that

$$
\begin{aligned}
& t^{\prime \prime}\left(\bar{c}_{1}, 1\right)=1 \\
& t^{\prime \prime}\left(\bar{c}_{1}, 0\right)=0 \\
& t^{\prime \prime}\left(\bar{c}_{i}, 0\right)=1 \quad \text { for } i \neq l(2 \leq i \leq n) .
\end{aligned}
$$

One can check that the term operation

$$
t(\bar{x}, z)=t^{\prime}\left(\bar{x}, t^{\prime \prime}(\bar{x}, z)\right)
$$

satisfies the requirements for $I$. Indeed,

$$
\begin{aligned}
& t\left(\bar{c}_{1}, 1\right)=t^{\prime}\left(\bar{c}_{1}, t^{\prime \prime}\left(\bar{c}_{1}, 1\right)\right)=t^{\prime}\left(\bar{c}_{1}, 1\right)=1 \\
& t\left(\bar{c}_{1}, 0\right)=t^{\prime}\left(\bar{c}_{1}, t^{\prime \prime}\left(\bar{c}_{1}, 0\right)\right)=t^{\prime}\left(\bar{c}_{1}, 0\right)=0, \\
& t\left(\bar{c}_{i}, 0\right)=t^{\prime}\left(\bar{c}_{i}, t^{\prime \prime}\left(\bar{c}_{i}, 0\right)\right)=t^{\prime}\left(\bar{c}_{i}, 1\right)=1 \text { for } i \neq l(2 \leq i \leq n),
\end{aligned}
$$

and

$$
t\left(\bar{c}_{l}, 0\right)=t^{\prime}\left(\bar{c}_{l}, t^{\prime \prime}\left(\bar{c}_{l}, 0\right)\right) \geq t^{\prime}\left(\bar{c}_{l}, 0\right)=1
$$

whence $t\left(\bar{c}_{l}, 0\right)=1$. This completes the proof.

Lemma 4.7. If $\mathbf{A}$ is a $G^{0}$-algebra of type $\mathbf{5}$ such that the variety $\mathrm{V}(\mathbf{A})$ is residually small, then for every term operation $f$ of $\mathbf{A}$, all $\widetilde{f}^{(j)}$ are term operations of $\mathbf{A}$.

Proof. Suppose that $\mathbf{A}$ is a $G^{0}$-algebra of type $\mathbf{5}$ such that the variety $\mathrm{V}(\mathbf{A})$ is residually small, and let $f$ be any $k$-ary term operation of $\mathbf{A}$ with canonical form (4.1). By symmetry it suffices to show that $\widetilde{f}^{(1)}$ is a term operation of $\mathbf{A}$. We will prove that if $t$ is a $(k+1)$-ary term operation whose existence is ensured by Lemma 4.6, then the term operation $t^{*}$ of $\mathbf{A}$ defined as

$$
t^{*}(\bar{x}, z)=f(\bar{x}) \wedge t\left(\bar{x}, z \wedge \wedge \bar{c}_{1}^{-1} \bar{x}\right)
$$

equals $\tilde{f}^{(1)}$.

In view of Lemma 2.2 , it suffices to verify that $\operatorname{Min}\left(t^{*}\right)=\operatorname{Min}\left(\widetilde{f}^{(1)}\right)$. Looking at the canonical form of $\widetilde{f}^{(1)}$ we can read off the elements of $\operatorname{Min}\left(\widetilde{f}^{(1)}\right)$ : they are the 
$(k+1)$-tuples $\left(\bar{c}_{1}, 1\right)$ and $\left(\bar{c}_{i}, 0\right)$ for $2 \leq i \leq m$. Thus we have to prove the following equality:

$$
\operatorname{Min}\left(t^{*}\right)=\left\{\left(\bar{c}_{1}, 1\right),\left(\bar{c}_{2}, 0\right), \ldots,\left(\bar{c}_{m}, 0\right)\right\} .
$$

Let $(\bar{c}, a) \in \operatorname{Min}\left(t^{*}\right)$. Then

$$
1=t^{*}(\bar{c}, a) \leq f(\bar{c}),
$$

so $f(\bar{c})=1$, and hence $\bar{c} \geq \bar{c}_{i}$ for some $i(1 \leq i \leq m)$. This implies that $(\bar{c}, a) \geq$ $\left(\bar{c}_{i}, 0\right)$. If $i \neq 1$, then we have

$$
t^{*}\left(\bar{c}_{i}, 0\right)=f\left(\bar{c}_{i}\right) \wedge t\left(\bar{c}_{i}, 0\right)=1 \wedge 1=1,
$$

therefore by the minimality of the tuple $(\bar{c}, a)$ we must have that $(\bar{c}, a)=\left(\bar{c}_{i}, 0\right)$. Now assume that $i=1$. Then

$$
1=t^{*}\left(\bar{c}_{1}, a\right) \leq t\left(\bar{c}_{1}, a \wedge \wedge \bar{c}_{1}^{-1} \bar{c}_{1}\right)=t\left(\bar{c}_{1}, a \wedge 1\right),
$$

so $t\left(\bar{c}_{1}, 1 \wedge a\right)=1$. In case $a \neq 1$ this would imply that $t\left(\bar{c}_{1}, 0\right)=1$ which contradicts one of the properties of $t$. Thus $a=1$, whence $(\bar{c}, a) \geq\left(\bar{c}_{1}, 1\right)$. Since we have

$$
t^{*}\left(\bar{c}_{1}, 1\right)=f\left(\bar{c}_{1}\right) \wedge t\left(\bar{c}_{1}, 1 \wedge \wedge \bar{c}_{1}^{-1} \bar{c}_{1}\right)=1 \wedge t\left(\bar{c}_{1}, 1\right)=1 \wedge 1=1,
$$

therefore again by the minimality of the tuple $(\bar{c}, a)$ we conclude that $(\bar{c}, a)=\left(\bar{c}_{1}, 1\right)$. This proves the inclusion $\subseteq$ in (4.4).

The equalities (4.6) and (4.5) show that $t^{*}$ assumes the value 1 for every $(k+1)$ tuple appearing on the right hand side of (4.4). Since these tuples are pairwise incomparable, they must all belong to $\operatorname{Min}\left(t^{*}\right)$. This completes the proof of the lemma.

\section{REFERENCES}

[1] D. Hobby and R. McKenzie, The Structure of Finite Algebras, Contemporary Mathematics v. 76, American Mathematical Society, 1988.

[2] K. A. Kearnes, E. W. Kiss, M. Valeriote, Minimal sets and varieties, Trans. Amer. Math. Soc. 350 (1998), 1-41.

[3] K. A. Kearnes, E. W. Kiss, M. Valeriote, A geometric consequence of residual smallness, to appear in Ann. Pure Appl. Logic.

[4] K. A. Kearnes, R. N. McKenzie, Residual smallness relativized to congruence types, Manuscript, 1996.

[5] K. A. Kearnes and Á. Szendrei, Self-rectangulating varieties of type 5, Internat. J. Algebra Comput. 7 (1997), 511-540.

[6] K. A. Kearnes and Á. Szendrei, A characterization of minimal locally finite varieties, Trans. Amer. Math. Soc. 349 (1997), 1749-1768.

[7] R. McKenzie, The residual bounds of finite algebras, Internat. J. Algebra Comput. 6 (1996), $1-28$.

[8] R. McKenzie, Recursive inseparability for residual bounds of finite algebras, Manuscript, 1995.

[9] Á. Szendrei, Term minimal algebras, Algebra Universalis 32 (1994), 439-477.

[10] Á. Szendrei, Maximal non-affine reducts of simple affine algebras, Algebra Universalis 34 (1995), 144-174. 
[11] Á. Szendrei, Strongly Abelian minimal varieties, Acta Sci. Math. (Szeged), 59 (1994), 25-42.

[12] R. Willard, A finite basis theorem for residually finite, congruence meet-semidistributive varieties, to appear in J. Symbolic Logic.

(Keith A. Kearnes) Department of Mathematics, University of Louisville, Louisville, KY 40292, USA.

E-mail address: kearnes@louisville.edu

(Ágnes Szendrei) Bolyai Institute, Aradi vértanúk tere 1, H-6720 Szeged, Hungary.

E-mail address: a.szendrei@math.u-szeged.hu 